\title{
On Indicators Oecd Proposes for Gauging Science \& Technology
}

\author{
Mario De Marchi \\ CNR-IRCRES, Consiglio Nazionale delle Ricerche, Istituto di Ricerca sulla Crescita Economica Sostenibile, via dei Taurini, Roma, Italy \\ Email: mario.demarchi@hotmail.com
}

How to cite this paper: De Marchi, M. (2019) Indicators Oecd Proposes for Gauging Science \& Technology. American Journal of Industrial and Business Management, 9, 2078-2082.

https://doi.org/10.4236/ajibm.2019.911137

Received: January 25, 2019

Accepted: November 26, 2019

Published: November 29, 2019

Copyright (c) 2019 by author(s) and Scientific Research Publishing Inc. This work is licensed under the Creative Commons Attribution International License (CC BY 4.0).

http://creativecommons.org/licenses/by/4.0/

(c) (i) Open Access

\begin{abstract}
The great number of theories of technological change witnesses a difficulty in devising and performing the crucial experiments which would allow a rational choice among alternative explanations. A preliminary step of the methodological process that must be completed to overcome such difficulty is the construction of consistent taxonomies of the S\&T Indicators available to test current theories. By rationally defining sound criteria for the measurement of S\&T activities, a well-founded taxonomy like that will provide scholars with clearer and better analytical instruments for theoretical discussion. As a consequence, the empirical investigation, which always ought to be closely linked with theory, could improve as well.
\end{abstract}

\section{Keywords}

Science \& Technology Indicators, Oecd

\section{Structure of the Article}

The article is organised as follows. In Section 2, the reasons for writing it, its main topics, its possible utility for Innovation Economics are discussed. Section 3 briefly recalls a previous article of which the paper is a development. Section 4 refers to some fundamental characteristics of Science and Technology in order to define criteria generating the proposed new taxonomy of Oecd S\&T Indicators. In Section 5, the essential features specifying each one of eight categories of Indicators, which make up our original taxonomy, are presented. Finally, in Section 6, perspectives for new research are hinted at.

\section{Introduction}

By accelerating the selection of the best methods and ideas for the scholars' dis- 
cussion to conceive, measure, and test S\&T indicators, a new original taxonomy of such indicators (which are collected worldwide applying Oecd standards) may strongly favour the progress in the measurement of scientific research and technological innovation activities. Indeed, just the great number of competing theories of technological change witnesses a difficulty in devising and performing the crucial experiments, which would allow a rational choice among alternative explanations. There is no scarcity of quantitative indicators suggested for the collection of statistical information on scientific research and technological innovation: far from it [1]. However, in the end, the very plenty of magnitudes may spoil the coherence of theoretical constructions, and it must be reined in by a rigorous set of rules. This article is an attempt at putting forth these rules by defining a consistent and exhaustive taxonomy of Oecd S\&T indicators, based first of all on a coherent description of the possible ways undertaken by rational search for new knowledge, involving either scientific research or technological innovation. The proposed taxonomy, if valid, would help to bring more order in the measurement of technological change and scientific progress. If this article succeeds in better clarifying the theoretical bases for S\&T measurement, then it will also contribute to improving the methods and analytical tools for empirical studies on the topic, given the pervasive link that in science always exists between abstract discussion and practical investigation.

\section{A First Attempt}

In an initial step towards a, much needed, consistent taxonomy of S\&T indicators, we drew the distinction which opposed indicators measured thank to "subjective statements" on S\&T activities against indicators resulting from "inter-subjective statements" (the latter referring to those assertions which derive from agreements among the opinions of scientists, technicians, entrepreneurs, etc.). Besides, a difference exists opposing "theoretical" investigation against "practical" activities. A couple of criteria were therefore based upon these contrasts regarding S\&T through: i) judgments on it (either subjective or inter-subjective) and ii) nature of its activities (either theoretical or practical). These criteria determine a four-category taxonomy of S\&T indicators [2] whose classes were identified by the combinations among the two couples of basic features attributed to Scientific and Technological activities. Further reflections have led its Author to abandon this taxonomy, which proved as tentative as most results always are in science's progress. On one side, the distinction between subjective and inter-subjective, although appealing at first sight, vanishes as one considers that for a judgement to enter accepted scientific knowledge it has to get some interpersonal approval, which makes "inter-subjective" every assertion about S\&T activities. On the other side, despite some questionable usages which may still be found in the literature, it is clear that the term "theoretical", concerning universal concepts, ought to be counterpoised to "ad-hoc" (explanation) not to "practical" [3]. Universal assertions with a practical content can well exist 
and they are found, for instance, in the field of applied research. As a consequence, the 4-category classification was abandoned, and replaced by a more sophisticated and complex taxonomy, one based on criteria that would hopefully not be constrained within too narrow epistemological approaches.

\section{Analyzing a Crucial Feature of S\&T}

In order to increase scope and relevance of our new taxonomy of Oecd's S\&T Indicators, we have based it on an irrefutably essential characteristic of S\&T activities: both the (abstract) science and the (practical) technology aim at reaching |REPRODUCIBLE-RESULTS|, and therefore consist in a search for |GENERAL-SOLUTIONS|. Three couples of twin characteristics can be derived from the |GENERAL-SOLUTIONS| binary relationship by simultaneously allowing for the |Science versus Technology| divide:

1) general versus particular;

2) abstract versus practical;

3) questions versus answers.

They determine eight categories. Let us examine in some detail each of them, using their qualifications to describe and label the different kinds of indicators told apart by our taxonomy.

\section{The Eight Categories}

\section{1) Contemplative science}

First comes the category of indicators on those S\&T activities which consist of the investigation of |general-abstract-questions|. The Oecd establishes clear bases to measure financial and human resources invested for this activity, that its Frascati Manual [4] defines as "Pure research".

2) Finalised research on general questions

The second category is made up by indicators measuring the S\&T activity specified by the triplet: |general-practical-questions|. Also, this category of indicators is neatly identified by the Frascati Manual, which provides scholars and statisticians with a safe basis for the measurement of "Applied research".

3) Scholarly literature

The third category of our taxonomy is formed by indicators measuring S\&T activities resulting in: |general-abstract-answers|. Scientists usually propose new solutions to the questions posed by their disciplines' research programmes, and submit them to their peers' scrutiny, by publishing them. Publication count is commonly considered a sensible way of weighing scientific output, provided that such measurement concerns large aggregates, which will tend to level out relevant qualitative differences among each article and among each quotation.

\section{4) Technological innovations}

Our fourth category is made up by indicators measuring the S\&T activities, which have resulted in: |general-practical-answers|. These consist of indicators measuring the introduction of new ways to meet general practical needs: namely 
technological innovations. About the statistical collection of the data, which produces such indices, Oecd's Oslo Manual [5] establishes sound, straightforward guidelines.

5) Experimental science

The fifth category of indicators includes those measuring S\&T activities which provide |particular-abstract-answers| by looking for new empirical observations. The progress that new evidence would, in the end, produce in a scientific discipline can prove impossible to foretell even for specialists. The indicators most plausibly attributed to Category (V) shall be inputs, such as the investment in the equipment needed to perform scientific experiments, and the wages paid to personnel involved in experimental research projects. Indeed, these proxies might sensibly estimate the relevance that, ex-ante, the scientific cadre and the policy-maker following its advice attribute to a particular abstract question.

6) Engineering

The sixth category of our taxonomy covers the indicators measuring S\&T activities that have produced |particular-practical-answers|. These indicators ought to gauge correctly the value of the new practical applications deriving from currently available theoretical knowledge. It is hard to estimate the precise value of this knowledge for the economic system or the society as a whole. Statistical offices will usually have to settle for a second best, by referring to a proxy such as the inputs in engineering activities.

7) Statistical induction

The seventh category is meant to include indicators measuring S\&T activities of researchers working on |particular-abstract-questions|. It consists of magnitudes estimating the value that the scientific community and the policy-maker attribute to the collection of data aimed at deriving general conclusions through their elaboration: for instance, human and financial inputs devoted to national statistical bureaux. The category is symmetrical to the "Experimental science" one. Indeed, in principle the activities classified in the "Experimental science" Category start with the conception (on the basis of whatever hint) of theories which actively rule the subsequent search for empirical evidence, that may refute or tentatively confirm the hypotheses. Conversely, the activities included in the "Statistical induction" Category begin with the collection of a (given) empirical evidence; then, attempts at generalising the properties shown by data are made, by using the methods of inductive Statistics.

\section{8) Finalised research on particular questions}

The last category is made up by indicators measuring the activity identified by the triplet |particular.practical-questions|: namely, data on resources invested in "Experimental development", following the Frascati Manual's definition of this search activity.

\section{Conclusions: Possible Applications of Our Taxonomy in Future Studies}

The criteria that our taxonomy adopts to sort out S\&T indicators is helpful to 
describe and explain the quantitative levels of S\&T activities in national innovation systems. Indeed S\&T indicators measure the initial circumstances assumed as data in these explanations and their predictions about the resulting S\&T activities. This is perhaps a trivial conclusion, but much less obvious is the further chance that our scheme offers to scholars: it allows them to make entirely new inferences, by going backwards along the path, which was originally followed to build the taxonomy. As a result of these logical steps, each of the indicators Innovation Theory utilises to gauge the S\&T activities could be disentangled according to the corresponding triplet identified by our taxonomy. And thanks to this subtler analysis of S\&T indicators scholars may reach a deeper understanding of the processes underlying scientific progress and technological innovation.

\section{Conflicts of Interest}

The author declares no conflicts of interest regarding the publication of this paper.

\section{References}

[1] Godin, B. (2004) Measurement and Statistics on Science and Technology: 1920 to the Present. Routledge, London. https://doi.org/10.4324/9780203481523

[2] De Marchi, M. (2016) A Taxonomy of S\&T Indicators, Scientometrics, 106, 1265-1268. https://doi.org/10.1007/s11192-015-1823-Z

[3] Popper, K.R. (1992) The Logic of Scientific Discovery. Routledge, London.

[4] Oecd (2015) Frascati Manual (Guidelines for Collecting and Reporting Data on Research and Experimental Development). Paris.

[5] Oecd (2018) Oslo Manual (The Measurement of Scientific, Technological and Innovation Activities). Paris. 